\title{
Why do scientific revolutions begin?
}

\section{By: Donald Gillies}

According to Kuhn's model, in most branches of science for most of the time the research scientists all accept the dominant paradigm of the field, and carry out normal science within the framework of that paradigm. Occasionally, however, a few of these research scientists challenge the dominant paradigm and try to develop a new one. If they are successful, we have a scientific revolution. The question I want to raise in this paper is why, in the midst of the usual normal science, do these occasional challenges to the dominant paradigm occur?

Kuhn himself provides an explanation which could be called the 'build-up of anomalies' view. On this account, if there are a large number of anomalies in the dominant paradigm, a few research scientists will begin to doubt its validity and look for another approach. I will begin the paper by criticizing this theory. One problem is that most paradigms most of the time have quite a number of anomalies, but this does not provoke a revolution. After all, the idea of normal science is to resolve anomalies, and a normal scientist can always take the attitude, which is often correct, that the existing anomalies will be resolved in due course. Moreover, there is little evidence that the number of anomalies is significantly greater in the period just before a scientific revolution begins than at other times. Indeed, in some cases, it looks as if the number of anomalies is less than usual just before the revolution. There is Kelvin's famous remark in 1900 (quoted by Lakatos) that 'the only cloud in the clear sky of the [ether] theory was the null result of the Michelson-Morley experiment.' Moreover the Michelson-Morley experiment had taken place 13 years before in 1887, and had been explained by the Fitzgerald-Lorentz contraction $(1893,1895)$. It looks as if the Einsteinian revolution of 1905 began in a situation of rather few anomalies.

In the paper I will argue in favour of a different theory of why scientific revolutions begin. This depends on taken account of technology and practical applications (or 'tech' for short). I distinguish two cases.

Case 1 will be called 'tech first'. Here an advance in the technology and practice of observation and experiment brings to light a hitherto unknown range of phenomena. The classic case of this did not occur at the beginning of a scientific revolution, but gave a boost to a revolution already under way. I have in mind the invention of the telescope and its use by Galileo to observe the heavens. In just a few nights of observation with the new technology, Galileo discovered the moons of Jupiter, mountains on the Moon, and added 80 new stars adjacent to the 9 known ones in Orion. Now it was actually not so difficult to accommodate these new discoveries within the Ptolemaic paradigm, but such striking and hitherto unknown phenomena 2 produced a new mental attitude - the feeling that these new phenomena required a new paradigm. My example of a 'tech first' scientific revolution will be the chemical revolution, which was stimulated by the new experimental techniques (e.g. the pneumatic trough, first with water and then with mercury) which enabled a number of new gases (elastic fluids) to be isolated, and their properties studied.

Case 2 will be called 'tech last'. Whereas, in case 1, the technology and practical developments come first, and act as an efficient cause of the scientific revolution, in case 2, there is an important technological and practical problem which cannot be solved within the old paradigm. This encourages some researchers to try out a new approach in the hope that it will enable the problem to be solved. So here the development in technology and practice is the final cause rather than the efficient cause. 
The example which will be given to illustrate this, is the revolution in medicine which led to the germ theory of disease and antiseptic surgery. This was begun by Lister while he was engaged in studying how to combat wound suppuration and hospital diseases. At the time the standard explanation of these phenomena was the miasma theory - the view that suppuration and disease were caused by bad air. This theory had recently been very successful as it lay behind Chadwick's programme for sewage disposal through building sewers, and Florence Nightingale's insistence on cleanliness in hospitals. These developments had certainly reduced disease. Moreover, chemistry had in the preceding decades made enormous advances, so that a chemical explanation of disease in terms of gases seemed an attractive scientific approach. However, the miasma theory made it almost impossible to devise a way of combating wound suppuration, since it was almost impossible to prevent air entering most wounds. This was not an in itself an anomaly in the Miasma theory, since the prevention of wound suppuration and hospital diseases might really have been very difficult indeed. Lister, however, after studying Pasteur's work, concluded that the cause of wound suppuration was not air but the microbes in the air. This new approach enabled him to solve the problem of wound suppuration using antiseptic surgery.

While in the paper I will concentrate on the two examples just described, if there is time, or in the discussion, I can outline how the tech first or tech last theory applies to other scientific revolutions, such as the Copernican revolution, or the Einsteinian revolution. 